\title{
The effect of the pelvis position in the sagittal plane on loads in the human musculoskeletal system
}

\author{
ROBERT MICHNIK ${ }^{1 *}$, HANNA ZADOŃ ${ }^{1}$, KATARZYNA NOWAKOWSKA-LIPIEC $^{1}$, \\ KATARZYNA JOCHYMCZYK-WOŹNIAK ${ }^{1}$, ANDRZEJ MYŚLIWIEC ${ }^{2}$, ANDRZEJ W. MITAS ${ }^{3}$ \\ ${ }^{1}$ Department of Biomechatronics, Faculty of Biomedical Engineering, Silesian University of Technology, Zabrze, Poland. \\ ${ }^{2}$ Institute of Physiotherapy and Health Science, Academy of Physical Education in Katowice, Katowice, Poland. \\ ${ }^{3}$ Department of Informatics and Medical Devices, Faculty of Biomedical Engineering Silesian University of Technology, \\ Zabrze, Poland.
}

\begin{abstract}
Purpose: The research work aimed to perform the mathematical modelling-based assessment concerning the effect of the position of the pelvis in the sagittal plane on loads present in the musculoskeletal system in the standing position. Methods: The analysis of the effect of various positions of the pelvis was performed using the Free Posture Model in the AnyBody Modeling System software. Simulated positions involving various values of pelvis inclination ranged from the extreme pelvic retroposition $\left(-7^{\circ}\right)$ through normative values $\left(0-23^{\circ}\right)$ to the extreme pelvic anteversion $\left(33^{\circ}\right)$. Results: The lowest resultant reaction forces in the intervertebral joints recorded for an angle of inclination restricted within the range of $9-27^{\circ}$ and segment L5-S1 amounted to less than $0.7 \mathrm{BW}$. A change in the pelvic inclination from the normative values towards retroposition or anteversion resulted in the increased muscular activity of the erector spinae, transverse abdominal muscles as well as internal and external oblique muscles. Regarding the lower limbs, changes in the activity were observed in the biceps femoris muscle, iliac muscle, gluteus minimus, gluteus medius and the gluteus maximus. Conclusion: The results obtained in the research-related tests confirmed that the pelvic inclination affects loads present in the musculoskeletal system. The abovenamed results will be used to develop therapeutic exercises aimed to reduce loads present in the musculoskeletal system. The aforesaid exercises will be used to teach participants how to properly position their pelvis and how to activate individual groups of muscles.
\end{abstract}

Key words: mathematical modeling, Anybody Modeling System, loads, lumbo-pelvic-hip complex, physiotherapy, physiopromotion

\section{Introduction}

Recent years have seen a significant increase in the number of diagnosed and treated back pain cases. Presently, in addition to hypertension and diabetes, the low back pain (LBP) is rated among civilisation diseases. As can be seen, the LBP is becoming not only health-related but also a social and economic problem. It is expected that approximately $50-80 \%$ of the society will suffer from the low back pain during the entire life span [9]. Presently, in Poland as many as $28 \%$ of women and $20 \%$ of men suffer from the LBP [18]. Statistical data published by the National Social Insurance Office (ZUS) state that in 2018 the low back pain was one of the most common reasons for inability to work and sick leaves [27].

The primary element of the physical examination of persons suffering from LBP is the observation of the posture and lumbopelvic complex movements. Related tests revealed that in people suffering from the low back pain it is possible to observe various and variable patterns of the lumbopelvic rhythm, potentially leading to the appearance and development of the LBP [20]. Because of this, to understand reasons for the presence of pain in the lumbosacral segment of the spine, it is necessary to take into consideration the functioning of the spine within the entire body, particularly the pelvis and lower limbs [12]. Changed motion patterns of the lumbopelvic complex trigger

\footnotetext{
* Corresponding author: Robert Michnik, Department of Biomechatronics, Silesian University of Technology, ul. Roosevelta 40, 41-800 Zabrze, Poland. Phone: +48 502041 426, e-mail: Robert.Michnik@polsl.pl

Received: March 27th, 2020

Accepted for publication: May 18th, 2020
} 
changes in the distribution of muscular tensions in the lumbar segment and in the rear vertebral ligaments. The necessity of analysing the entire complex is confirmed by symptoms present in persons suffering from the LBP [12] including the improper positioning of the pelvis (usually anteversion), the weakening of gluteal muscles, sciatic-tibial muscle tension, increased iliopsoas and quadratus lumborum tension. The key aspect of preventing the development of pain is effective prevention. In terms of the LBP prevention, particular attention should be paid to exercises stabilising the lumbar segment of the spine. The purpose of such exercises include the control and coordination of paravertebral muscles to maintain the dynamic stability of the spine without compromising the proper posture [19].

Presently, the lumbopelvic hip complex in patients (LPHC, i.e., the position of the pelvis and the determination of the spinal curvatures) is assessed using such tools as inclinometer [4], duometry [4] and measurements of X-ray images [6]. Primarily, the above-named methods enable measurements of the inclination of spine curvatures or that of the pelvis, yet they do not enable the analysis of loads present within the musculoskeletal system. Little is still known about the effect of the individual elements of the lumbopelvic hip complex on load values. Numerous research works indicate the necessity of gaining detailed knowledge about lumbopelvic movements both in the LBP prevention and in the development of a diagnostic therapy [1], [10], [17]. A method enabling the identification of the correlation between the kinematics of movement and generated loads of the locomotor system is the mathematical modelling of the human musculoskeletal system involving the use of static or dynamic optimisation [1], [5], [8], [14]-[16], [24]-[26]. The greatest advantage resulting from the application of mathematical models is the possibility of non-invasive obtainment of information about, among other things, reaction forces in joints and muscular forces. The increasing need for the determination of the mechanisms behind the generation of loads and the analysis of generated loads, both in the skeletal system and in the muscular system, contributed to the development of software programmes. Such programmes allow the development of mathematical models of the human locomotor system. A number of mathematical models enabling the performance of simulations, both in static and dynamic conditions, are available in the libraries of commercial environments such as OpenSim or AnyBody Modeling System. The aforesaid software programmes make it possible to use and modify models implemented in the environment as well as to cre- ate such models independently. Mathematical models of the human locomotor system available in the libraries of the above-named environments are described by means of the kinematic structure of the skeleton, parameters of mass-inertial elements, the number of muscles, locations of attachments of related muscles and the model of the dynamics of a given muscle. In turn, the identification of loads present in the musculoskeletal system consists in solving the inverse problem of dynamics and then the estimation of muscular force values using static optimisation.

The research work discussed in this article aimed to perform the mathematical modelling-based assessment of the effect of the pelvic inclination in the sagittal plane on the presence of loads in the musculoskeletal system in the standing position.

\section{Materials and methods}

Loads present in the human musculoskeletal system were determined through simulations performed in the AnyBody Modeling System environment (AnyBody Technology Inc., Aalborg, Denmark) and a model of the entire human body, i.e., the FreePosture Model (AnyBody Managed Model Respository, AMMR ver.1.5). The aforesaid model is a static model made up of 69 rigid bodies representing individual segments of the human body. The segments were connected by means of kinematic pairs characterised by the number of degrees of freedom dependent on anatomical conditions of individual joints. The spine model was built by single thorax segment involving ribs, cervical and thoracic vertebrae, lumbar section containing 5 individual vertebrae L1-L5, and sacrum, which was rigidly connected to the pelvis. Intervertebral joints were modelled as rigid bodies joined by spherical joints creating kinematic pairs of 3 degrees of freedom. This kind of joints can provide reaction forces but not reaction moments [2], [25]. Ligaments were not included in the model, so the model presumes no significant contribution to torque production from ligaments.

The muscular system was composed of approximately 1000 linear elements representing muscular actons. The action line of actons was defined using the initial and final point (or via-points) which a given muscle goes through. The maximum strength of individual muscle fibres was described using the product of the muscle cross-section and specific muscle tension [25]. The model utilised the default lumbar spine rhythm, which, according to publication [23], deter- 
mines the range of movements at various levels of the lumbar segment. The assumed rhythm is characterised by the increased participation of individual vertebrae in the movement of the entire lumbar segment along with the transition from the lower segment of the spine upwards. The mathematical model used in the tests was repeatedly subjected to verification based on the information obtained in experimental tests [2], [5], [25].

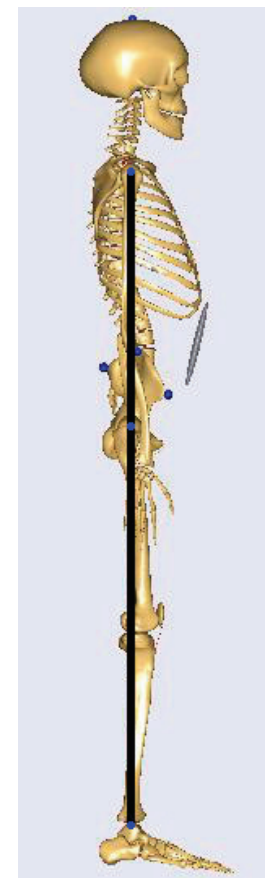

Fig. 1. Standing position - example
Input data used to develop the standing position in the AnyBody Modeling System environment included values of angles in the ankle, knee joint, hip joint, shoulder joint, cubital joint as well as the pelvic inclination angle and the angle of trunk inclination. The above-named angles were selected so that the ankle, hip joint and the shoulder joint could be in a straight line regardless of the pelvic inclination angle (Fig. 1). Simulations with an increment of $1^{\circ}$ were performed in relation to the pelvic inclination angle (defined as the angle between the straight line determined by the anterior superior iliac spines and the anterior inferior iliac spines and the horizontal straight line (Fig. 2)) restricted within

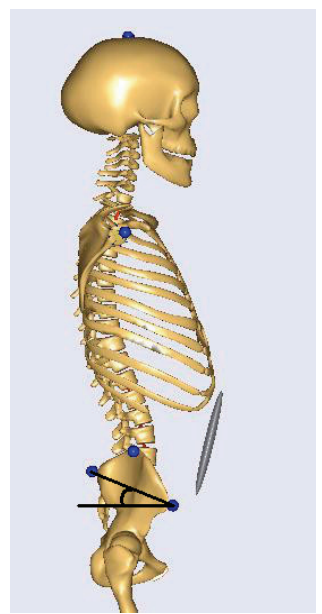

Fig. 2. FreePosture Model with the marked angle of pelvis inclination

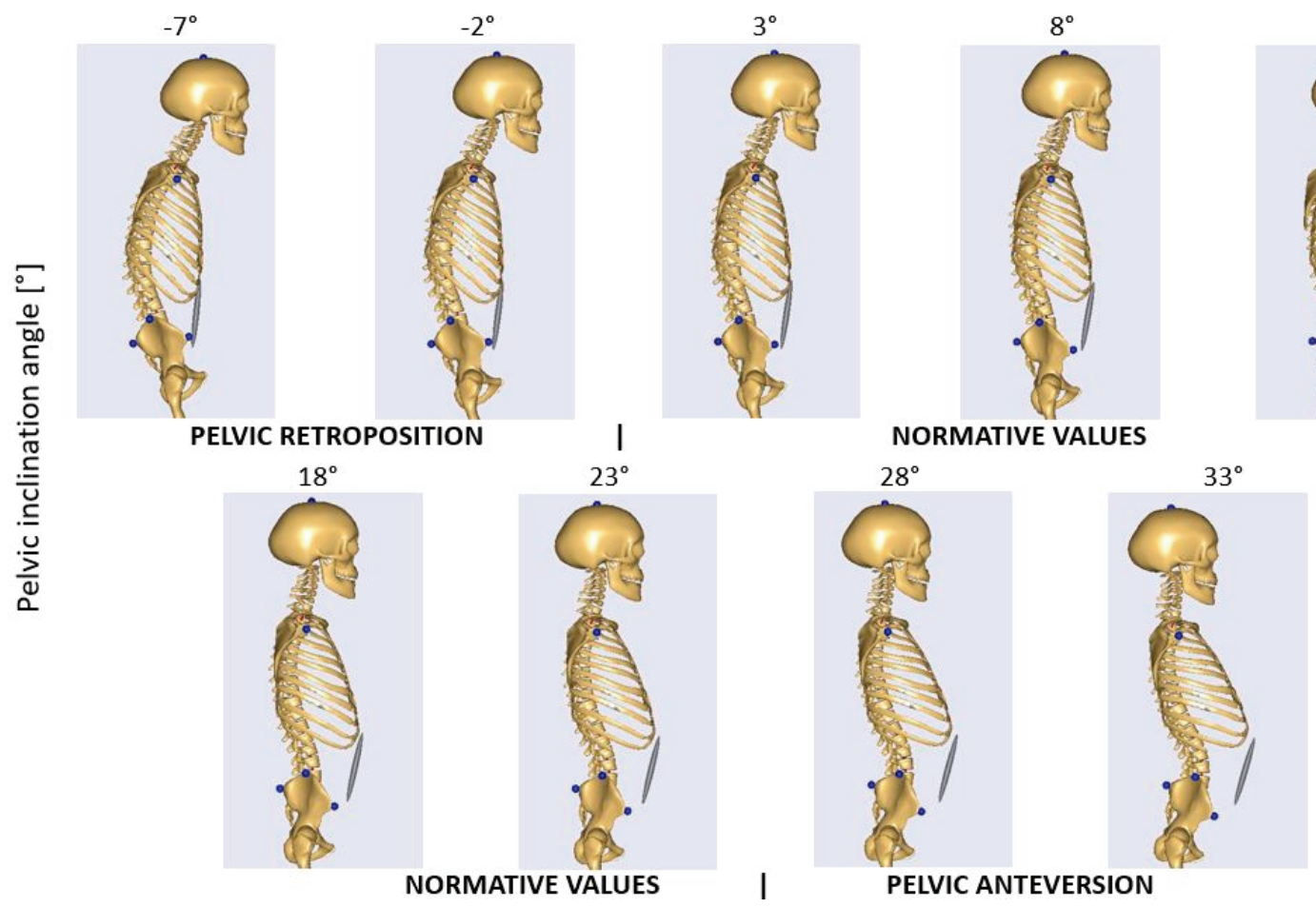

Fig. 3. Range of analysed movements 
the range of $-7^{\circ}$ to $33^{\circ}$ (Fig. 3). The aforesaid range was adopted on the basis of reference publications [4], where the normative pelvic inclination values were those of an angle restricted within the range of $0^{\circ}$ to $23^{\circ}$. In turn, the extreme values amounting to $-7^{\circ}$ (pelvic retroposition) and $33^{\circ}$ (pelvic anteversion) were adopted on the basis of the tests performed by Levine D. et al. [11], involving the determination of the average pelvic inclination values in relation to the neutral position during the maximum positioning of the pelvis in the anteversion and retroposition. During the adjustment of the FreePosture model, the following assumptions and simplifications were adopted:

- simulations were performed exclusively for the 50th percentile male model (height $-175 \mathrm{~cm}$, body weight $-75 \mathrm{~kg}$ ),

- it was assumed that the human body was symmetric,

- adopted muscular model was AnyMuscle Model, i.e., a simple model assuming the constant value of muscular force independent of muscle work conditions,

- ground reaction force was modelled using two vectors put against the heel and instep,

- standing position was modelled as the position where the ankle, hip joint and the shoulder joint were in one vertical line regardless of the pelvic inclination angle,

- simulations were performed for a pelvic inclination angle restricted within the range of $-7^{\circ}$ to $33^{\circ}$, using an increment of $1^{\circ}$.

In the AnyBody Modeling System environment, the identification of loads in the musculoskeletal system consists in the solving of the inverse problem of dynamics and the estimation of muscular force values using static optimisation. The adopted optimisation criterion was the criterion of movement control, assuming the minimisation of the sum of cubes of the proportion of muscular forces to maximum forces. The form of the optimisation problem was identified using the following dependences:

- objective function:

$$
\begin{gathered}
\min G\left(f^{(M)}\right), \\
G\left(f^{(M)}\right)=\sum_{i=1}^{n^{m}}\left(\frac{f_{i}^{(M)}}{N_{i}}\right)^{3}, \quad i=1, \ldots, n^{M},
\end{gathered}
$$

- limiting conditions:

$$
\begin{gathered}
\mathbf{C} f=d, \\
0 \leq f_{i}^{(M)} \leq N_{i}, \quad i \in 1, \ldots, n^{(M)},
\end{gathered}
$$

where:

$f^{(M)}-$ muscular force,

$N_{i}$ - maximum muscle force,

$n^{M}-$ number of muscles,

C - matrix of arms of muscular strength action and reactions in relation to a given joint,

$f$ - matrix of muscular forces and reactions present in the system,

$d$ - resultant moments of external forces determined in relation to a given joint.

The results obtained in the simulations enabled the analysis of the effect of the pelvic inclination on the following:

- reaction forces present in the intervertebral joints of the lumbar segment of the spine T12-L1, L1-L2, L2-L3, L3-L4, L4-L5, L5-S1,

- muscular activity of the muscles located within the lumbar segment, i.e., erector spinae, rectus abdominis muscle, transverse abdominal muscle, abdominal internal oblique muscles, abdominal external oblique muscles and multifidus muscle,

- activity of the muscles of the lower limbs.

The results were normalised in relation to the human body weight (BW).

\section{Results}

The performance of a number of simulations in the AnyBody Modeling System environment enabled the analysis of the effect of the position of the pelvis on the sagittal plane on values of reaction forces in the intervertebral joints of the lumbar segment of the spine and the activity of the muscles within the lumbar segment of the spine and the muscles of the lower limbs.

\subsection{Reactions in the intervertebral joints of the lumbar section of the spine}

Figure 4a presents changes in resultant reaction forces (the absolute value of the reaction force vector) in the intervertebral joints of the lumbar segment of the spine.

The simulation revealed that the greatest loads affected segment L5-S1 and, depending on a pelvic inclination angle, were restricted within the range of $1.03 \mathrm{BW}$ to $0.88 \mathrm{BW}$ in relation to the pelvic retroposition, $0.85 \mathrm{BW}$ to $0.65 \mathrm{BW}$ in relation to the normative values and $0.66 \mathrm{BW}$ to $0.76 \mathrm{BW}$ in relation to the 


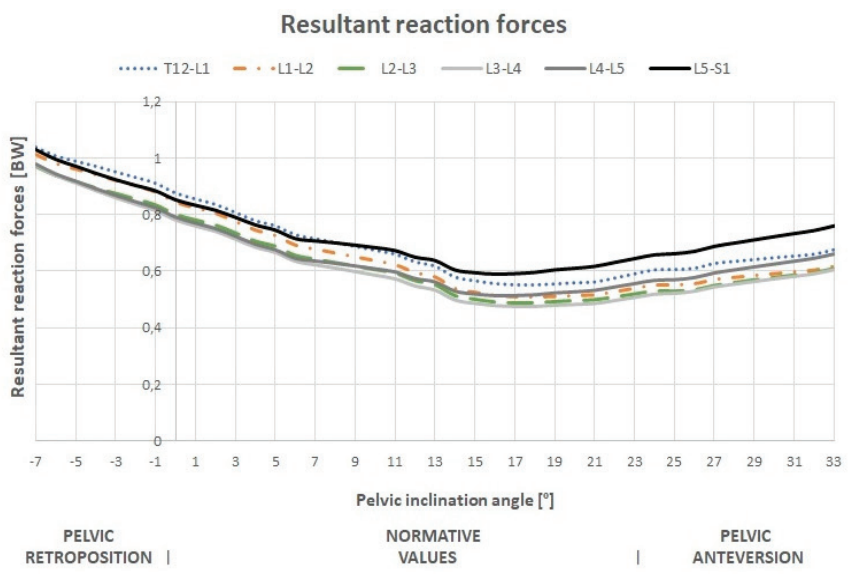

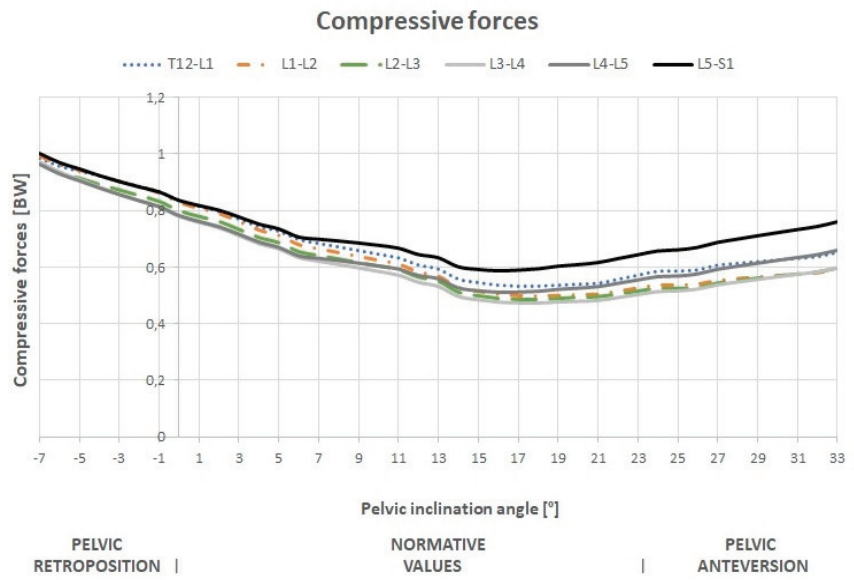

(b)

(a)

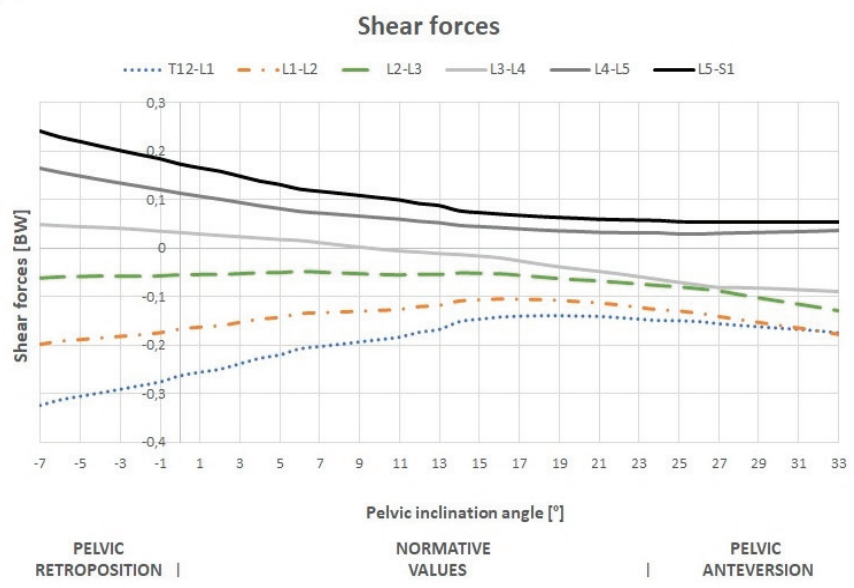

(c)

Fig. 4. Values of reaction forces present in the lumbar segment of the spine in relation to various positions of the pelvis in the sagittal plane: (a) resultant forces, (b) compressive forces, (c) shear forces

pelvic anteversion. The lowest value of reaction force (i.e., 0.59 BW) in segment L5-S1 was recorded in relation to a pelvic inclination angle of $16^{\circ}$. It was noticed that in relation to the pelvic inclination range of $9^{\circ}$ to $27^{\circ}$, the resultant values of reaction forces did not exceed $0.7 \mathrm{BW}$.

The analysis also included individual components of reaction forces in the intervertebral joints, i.e., compressive forces (proximo-distal force) and shear forces (antero-posterior force) (Figs. 4b, c). In terms of compressive forces, a change in the pelvic inclination (retroposition or anteversion) led to increased values of shear forces, compared to those accompanying the basic, i.e., normative, position. Values of shear forces decreased along with the change from the pelvic retroposition through the normative values to the pelvic anteversion. Only in relation to segment L3-L4, the exceeding of a pelvic inclination angle of $12^{\circ}$ triggered a change in the direction of force action and an increase in the value of force along with the deepening of the pelvic anteversion.

\subsection{Muscular forces}

The simulations performed in the AnyBody Modeling System environment enabled the analysis of the muscular activity of the muscles located within the lumbar segment of the spine and lower limbs. Figures 5 and 6 present the values of muscular forces in relation to various positions of the pelvis in the sagittal plane. The grey colour indicates the range within which the resultant reaction forces in segment L5-S1 amounted to less than $0.7 \mathrm{BW}$, i.e., the pelvic inclination angle range of $9^{\circ}$ to $27^{\circ}$. The black colour represents a pelvic inclination angle of $16^{\circ}$, corresponding to the lowest value of the resultant reaction force recorded in relation to segment L5-S1.

Among the analysed muscular activities of the muscles located within the lumbar section of the spine, i.e., the erector spinae, rectus abdominis muscle, transverse abdominal muscle, abdominal internal oblique muscles and abdominal external oblique muscles (Fig. 5), the 


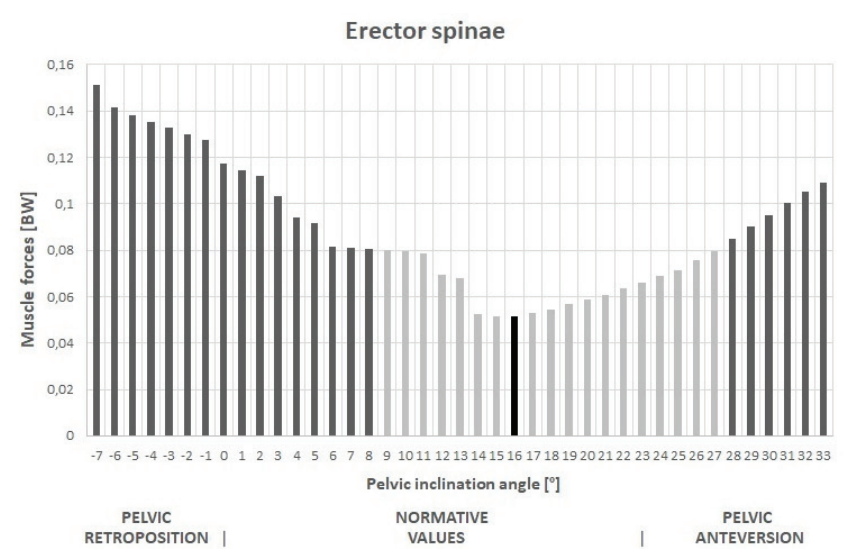

(a)

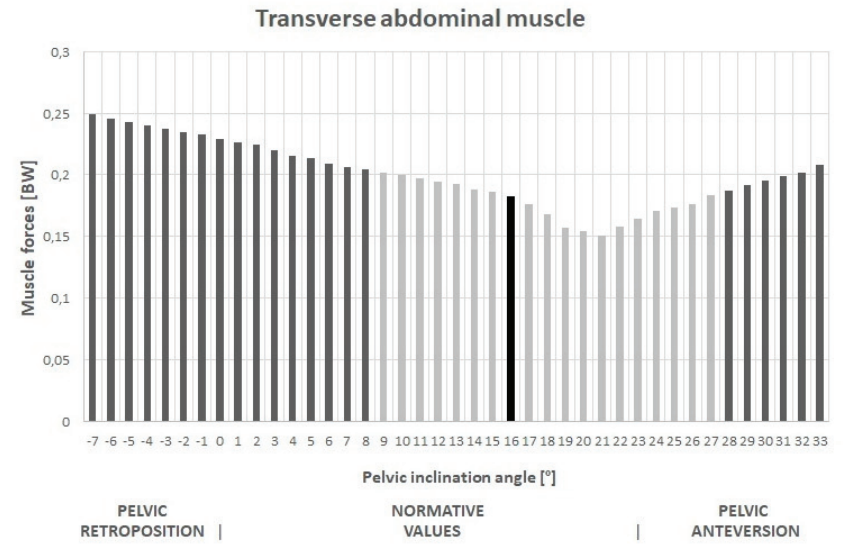

(c)

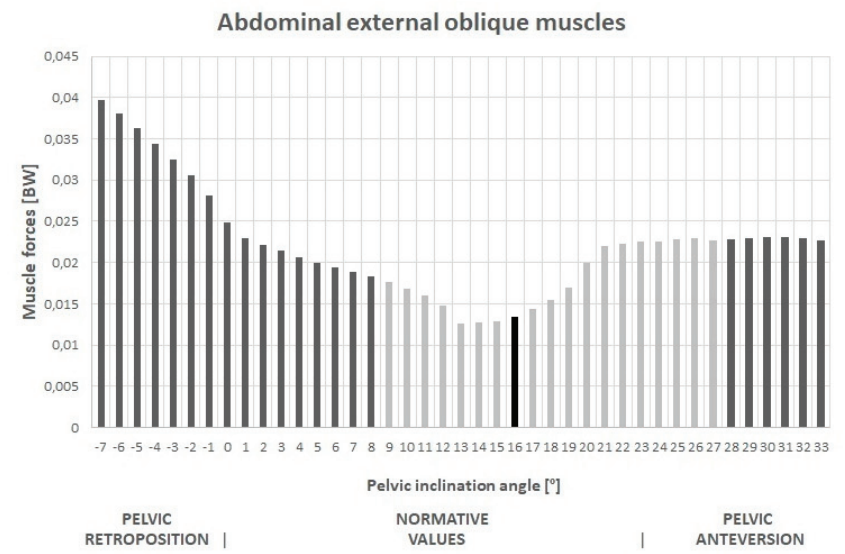

(e)

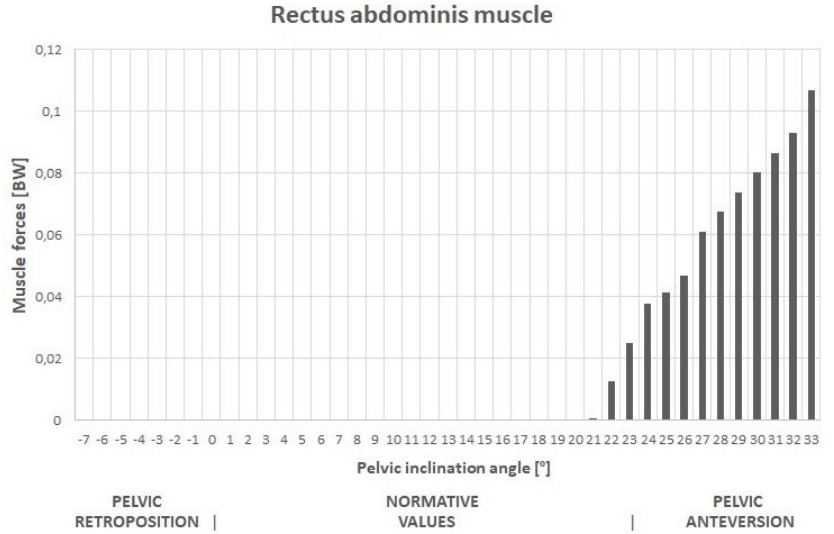

(b)

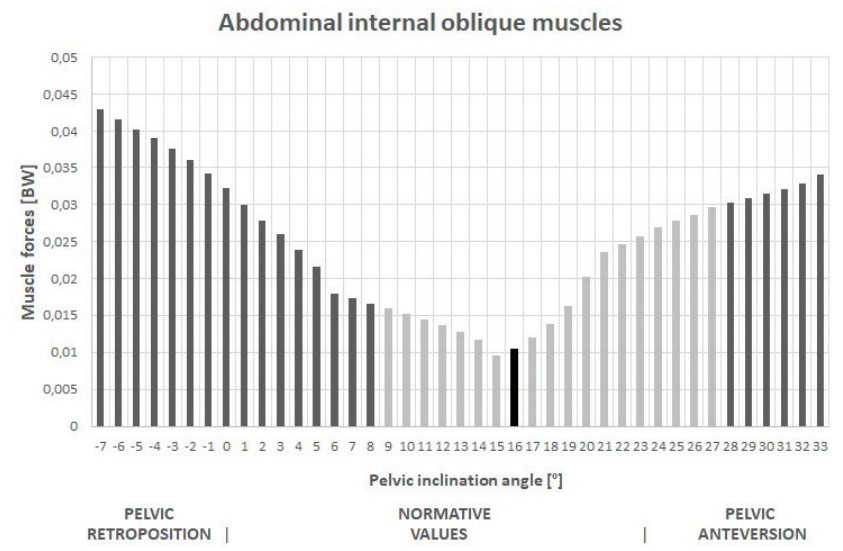

(d)

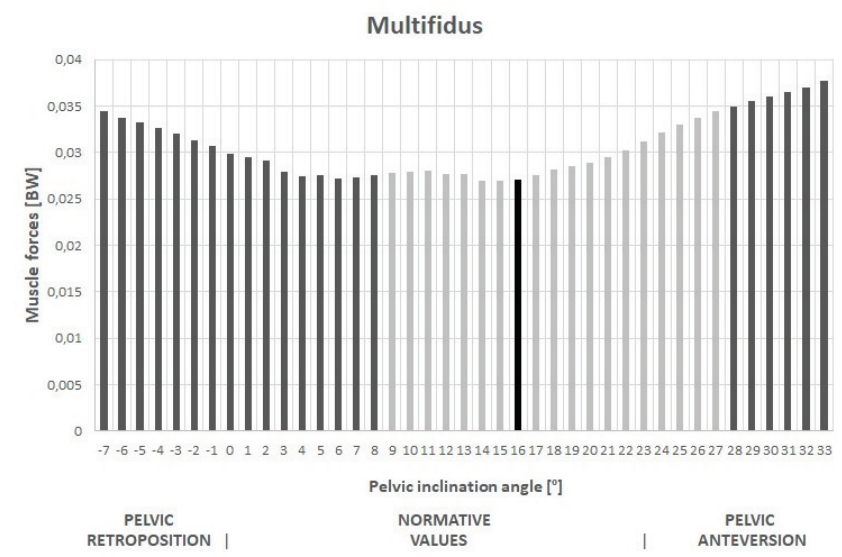

(f)

Fig. 5. Values of muscular forces of the muscles located within the lumbar segment: a) erector spinae, b) rectus abdominis muscle, c) transverse abdominal muscle, d) internal oblique muscle, e) external oblique muscle and f) multifidus

highest activity was that of the erector spinae. During the pelvic retroposition, the values of the muscular forces of the erector spinae were restricted within the range of $0.12 \mathrm{BW}$ to $0.15 \mathrm{BW}$ and decreased along with the increasing inclination of the pelvis forward to the obtainment of a pelvic anteversion angle of approximately $16^{\circ}$. After exceeding the aforesaid value, the activity of the erector spinae grew up to approximately $0.11 \mathrm{BW}$.
During the analysis assessing the effect of the pelvis position on the functioning of abdominal muscles simulations revealed that the activation of the rectus abdominis muscle (Fig. 5b) occurred after the pelvis reached its anteversion. The deepening of the pelvic anteversion was accompanied by an increase in the value of muscular forces. The activation of the muscle took place when the pelvic inclination angle reached approximately $22^{\circ}$. 
Figure $5 \mathrm{c}$ presents changes in the muscular force of the transverse abdominal muscle in relation to an angle of pelvis inclination. The highest activity was recorded in relation to the pelvic retroposition with values gradually decreasing along with a decrease in the pelvic inclination angle to $22^{\circ}$. The pelvic anteversion led to a repeated increase in muscular activity.

Both, in terms of abdominal internal oblique muscles and abdominal external oblique muscles
(Figs. 5d, e), the lowest muscular activity was observed in relation to a pelvic inclination angle restricted within the range of $13-15^{\circ}$. It was possible to observe the intensification of muscular activity when the pelvis was in the retroposition or anteversion. It was observed that the muscular activity of the abdominal external oblique muscles did not change and amounted to approximately $0.02 \mathrm{BW}$ in the pelvic anteversion.

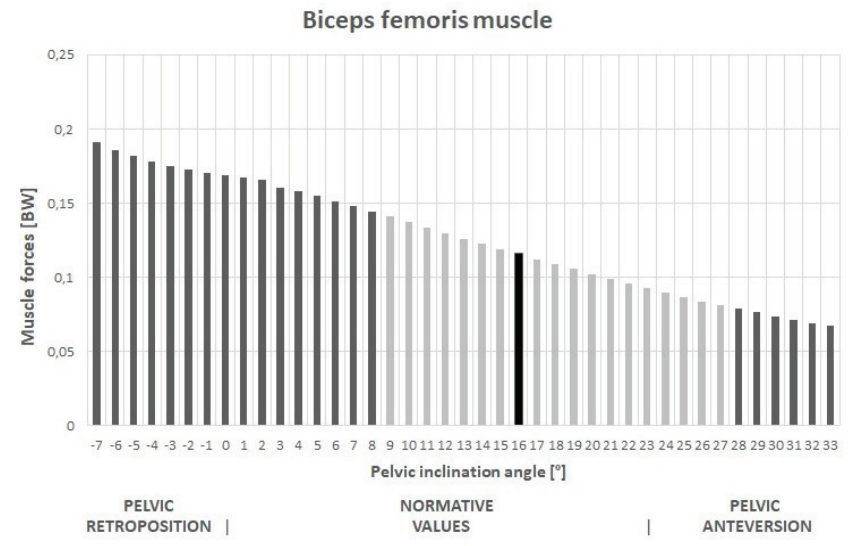

(a)

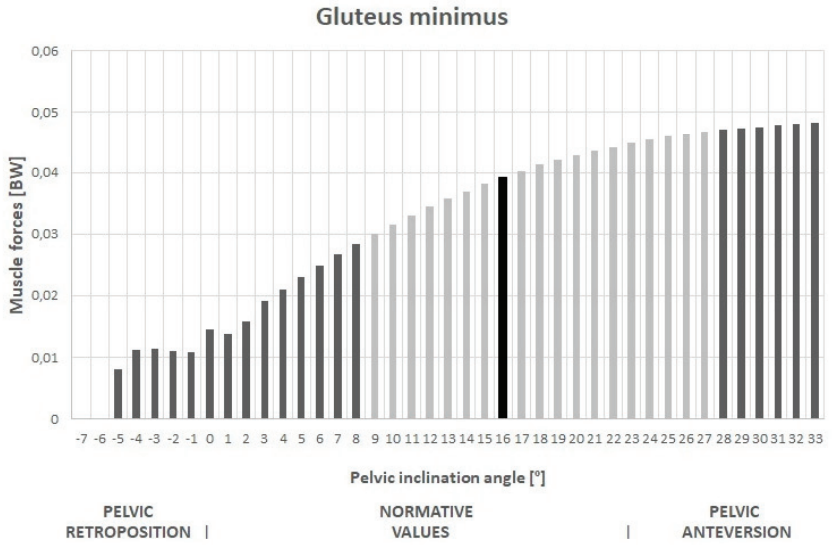

(c)

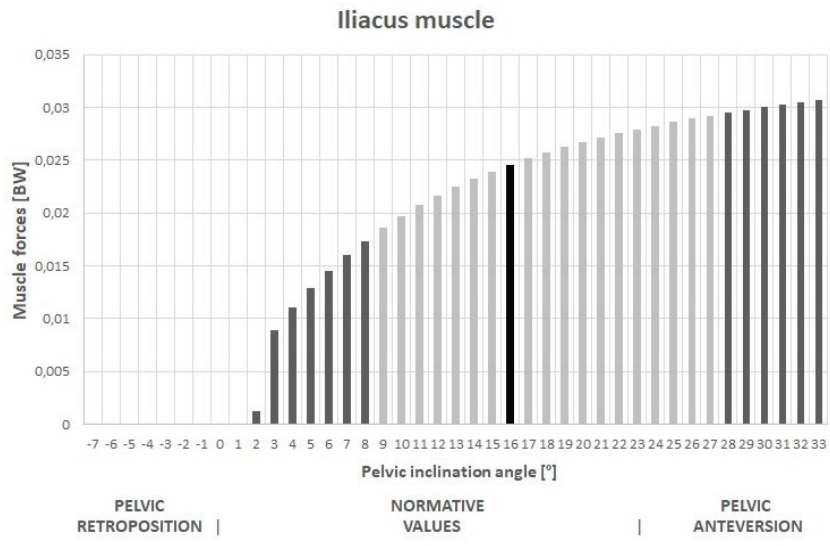

(b)

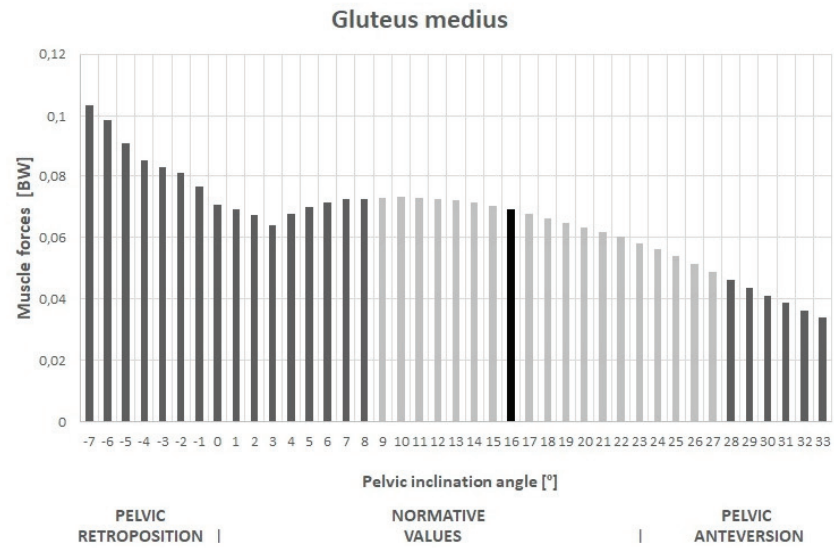

(d)

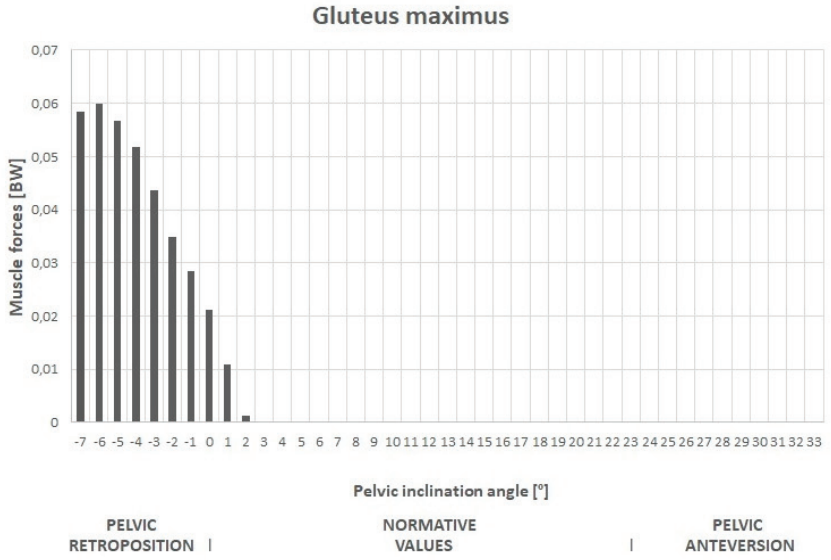

(e)

Fig. 6. Muscular activity of selected muscles of lower limbs: (a) biceps femoris muscle,

(b) iliac muscle, (c) gluteus minimus, (d) gluteus medius and (e) gluteus maximus 
Figure $5 \mathrm{f}$ shows changes in the muscular force of the multifidus muscle. The lowest activity was recorded in relation to a pelvic inclination angle of $15^{\circ}$. The deeping of the pelvic anteversion or retroposition increases muscular activity. In the case of pelvic retroposition, the activity was reduced again when the pelvic inclination angle reached approximately $4^{\circ}$.

The analysis also involved the effect of the pelvic position on the muscular activity of the muscles of the lower limbs. The results revealed that the standing posture was connected with the unchanged activity of the gastrocnemius muscle $(0.49 \mathrm{BW})$, tibialis posterior muscle $(0.32 \mathrm{BW})$, semitendinosus muscle $(0.14 \mathrm{BW})$ and the semimembranosus muscle $(0.15 \mathrm{BW})$. A change in the pelvic position alters the muscular activity of the biceps femoris muscle, iliac muscle, gluteus minimum, gluteus medius and gluteus maximus (Fig. 6).

The analysis of the results revealed that the muscular activity of the biceps femoris muscle and gluteus medius decreased along with the changing pelvic position from the retroposition through the normative values to the anteversion (Figs. 6a, d). In relation to the biceps femoris muscle the value of muscular force decreased from $0.19 \mathrm{BW}$ to $0.07 \mathrm{BW}$, whereas in relation to gluteus medius, the muscular force value decreased from $0.1 \mathrm{BW}$ to $0.03 \mathrm{BW}$. The opposite tendency could be observed as regards the iliac muscle and gluteus minus (Figs. 6b, c), where the value of muscular activity increased from 0 to $0.03 \mathrm{BW}$ (iliac muscle) and from 0 to $0.05 \mathrm{BW}$ (gluteus minus). It should be noted that in relation to pelvic retroposition the iliac muscle is deactivated whereas the muscular activity of gluteus maximus increases (Fig. 6e).

\section{Discussion}

The overloading of the spine is reported by an increasingly large number of people. The lower back pain affects not only selected occupational sectors but the majority of society. Reasons for the pain may include the overloading of the locomotor system resulting from the improper positioning of body segments, the insufficiency of the muscular system and motoric control disorder. This study, involving mathematical modelling methods, aimed to verify whether the position of the pelvis in the sagittal position affected loads present in the musculoskeletal system of the lumbar segment of the spine.

The simulations assessing the effect of pelvic position on spinopelvic parameters and performed in the AnyBody Modeling System environment confirmed the results of experimental tests [11] and of X-ray examinations involving healthy persons [6]. The simulations, experimental tests and X-ray examinations revealed that changes in the pelvic inclination affected the position of the spine in its lower parts, i.e., the lumbosacral segment (Fig. 7). The pelvic retroposition triggers the loss of natural lumbar curvature resulting in the "flattening" of the lordosis. In turn, the transition from pelvic retroposition through the normative posture to the pelvic anteversion leads to the protrusion of the lumbar lordosis.

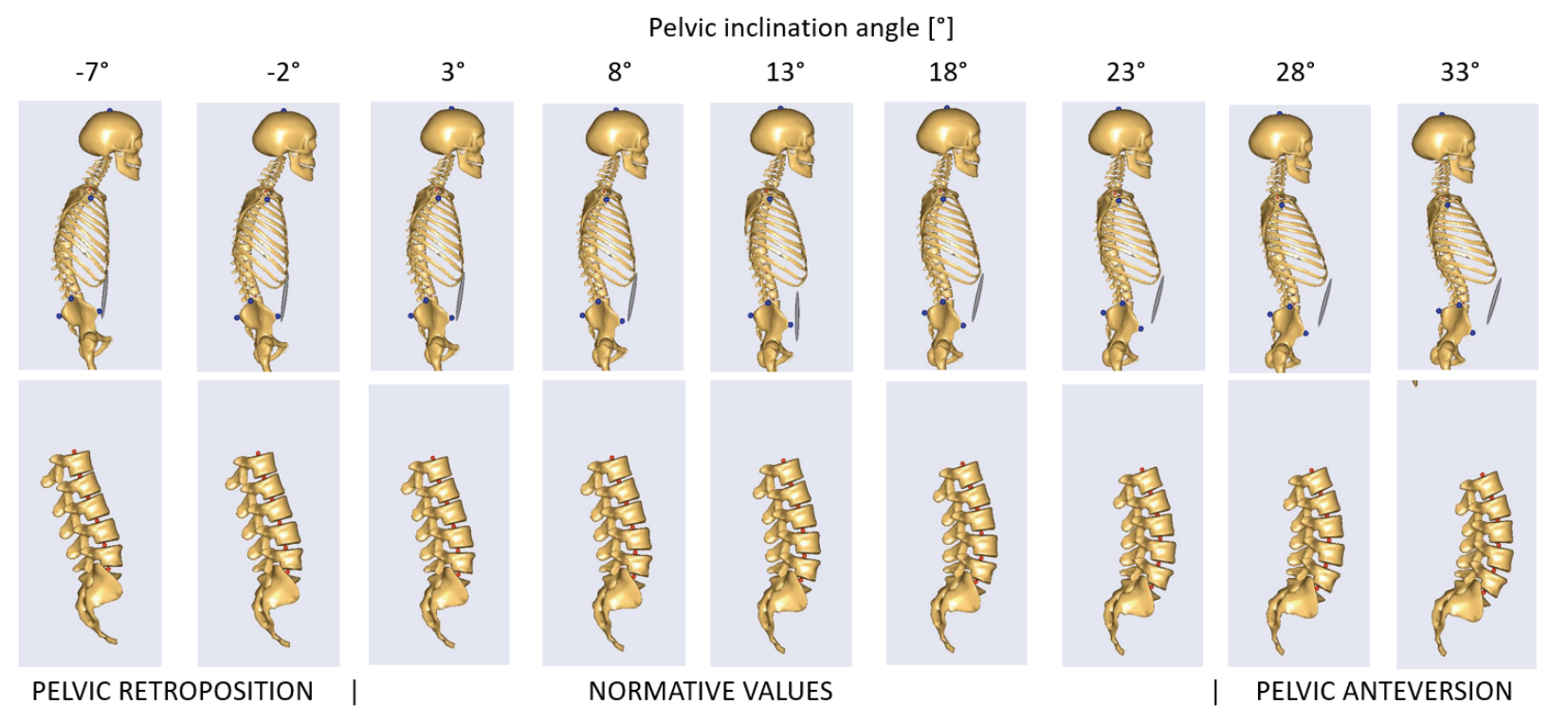

Fig. 7. Position of the lumbar segment in relation to the pelvis inclination angle 
The simulation demonstrated that the position of the spine had an impact on the values of reaction forces in the intervertebral joints of the lumbar segment of the spine. In actual conditions, the foregoing translates into the diversified distribution of loads affecting intervertebral discs. Scientific information concerning spinal biomechanics and results of computeraided simulations by Joszko et al. [7] and SzkodaPoliszuk et al. [21] justify the conclusion that the pelvic retroposition, imposing the straightening of the lower part of the lumbar segment of the spine, is responsible for the uniform loading of intervertebral discs and for the asymmetric loading of intervertebral discs in terms of the pelvic anteversion. In spite of the positive effect resulting from the straightening of the spine, it is necessary to pay attention to the generation of high values of reaction forces in the intervertebral joints being 1.5 times higher than those generated when the pelvic inclination angle amounted to $16^{\circ}$, whereas the lowest value $(0.59 \mathrm{BW})$ was obtained for segment L5-S1. Numerous tests revealed that the shape of the lumbar lordosis was also affected by age, sex, body weight, ethnic origin or sports activity [3], [13], which were not included in the above-presented model tests.

The above-presented results are inconsistent with conclusions contained in works by Hayden et al. [6] and Walker et al. [22] stating the lacking effect of the pelvic position on the lumbar lordosis and the functioning of abdominal muscles. The simulations revealed that the proper position of the pelvis was essential for the adoption of a proper bodily posture. The position of the pelvis not only affects the position of the lumbar segment of the spine and reaction forces in the intervertebral joints of the spine but also influences the functioning of the entire muscular system. The pelvic inclination activates various groups of muscles. The deepening of the pelvic anteversion or retroposition increases the muscular activity of the erector spinae, transverse abdominal muscles as well as abdominal internal and external oblique muscles. The muscles which are activated solely in the pelvic anteversion or retroposition are gluteus maximus and the rectus abdominis muscle.

The substantiated weakening of abdominal muscles in persons suffering from the LBP [6] implies that the strengthening of abdominal muscles through therapeutic exercises based on the rotation of the pelvis may pave the way for the prevention and rehabilitation capable of eliminating lower back pain. A particularly important role is played by musculus transversus abdominis and multifidus, which by improving the quality of central stabilisation, are decisive for the low-energy maintaining of the standing posture.
The results obtained in this paper can be considered reliable, although undoubtedly some limitations of the presented research and analyses can be indicated. Significant limitation is the fact that simulation involved isolated changes of pelvis tilt. The relationship between pelvic tilt and a change in the position of the torso or the centre of body mass was not studied. Moreover, in all simulations it was assumed that the ankle, hip and shoulder joints are in one line may be a limitation. A change in pelvic tilt may affect the position of the hip and shoulder joint relative to each other. However, taking the above information into account would require experimental testing on a large group of people with different pelvic positions. However, the testing methods and obtained simulation results presented in the paper extend knowledge concerning the sensitivity of the applied model to changes pelvis tilt.

The above-presented pilot research work involved the sole performance of modelling-based tests. It should be noted that the simulations were performed using a model of the entire human body representing a European male and an algorithm describing the lumbopelvic rhythm, pre-implemented in the Modeling System environment. The authors intend to continue the research by performing experimental tests constituting the basis for repeated static and dynamic simulations. Both the obtained test outcome and the results of tests to be performed in the future will be used to develop and verify therapeutic exercises aimed to teach/learn how to optimally position the pelvis in relation to the musculoskeletal system.

\section{Conclusions}

The research work aimed to identify the effect of the position of the pelvis in the sagittal plane on loads present in the musculoskeletal system. The analysis of the resultant reaction forces and muscular forces revealed that the pelvic inclination angle triggers changes in loads. The test results showed that a pelvic inclination restricted within the range of $9-27^{\circ}$ was accompanied by the lowest values (below $0.7 \mathrm{BW}$ ) of the resultant reaction forces in segment L5-S1. In addition to being responsible for the loss of the natural spinal curvature, the pelvic retroposition activates gluteus maximus. In turn, the pelvic anteversion activates the rectus abdominis muscle. A change in the pelvis inclination angle affects the activity of the erector spinae, abdominal muscles, multifidus muscle and muscles of lower limbs, i.e., among others, biceps femoris muscle, iliac muscle, gluteus minimus, gluteus medius and gluteus maximus. 


\section{Acknowledgement}

The study was realized within the project "DISC4SPINE dynamic individual stimulation and control for spine and posture interactive rehabilitation" (grant no. POIR.04.01.02-00-0082/17-00) co-founded by the European Regional Development Found within Operational Program Smart Growth Action 4.1.2.

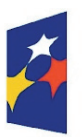
Funds Smart Growth
European

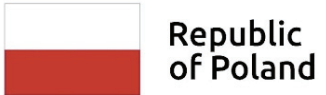

European Regional Development Fund
European Union

\section{References}

[1] Bassani T., Casaroli G., Galbusera F., Dependence of lumbar loads on spinopelvic sagittal alignment: An evaluation based on musculoskeletal modeling, PLoS One, 2019, 14, e0207997.

[2] Bassani T., Stucovitz E., Qian Z., Briguglio M., GALBUSERA F., Validation of the AnyBody full body musculoskeletal model in computing lumbar spine loads at L4L5 level, J. Biomech., 2017, 58, 89-96.

[3] Been E., Kalichman L., Lumbar lordosis, Spine J., 2014, 14, 87-97.

[4] BiBROwicz K., OsiŃSKa M., Characteristics and evaluation of the relationship between pelvic inclination in the sagittal plane and the size of the anterior-posterior curvature of the spine in schoolchildren aged 13, 14 and 15 years, Issues Rehabil. Orthop. Neurophysiol. Sport Promot. - IRONS, 2012, $1,66-74$

[5] Damsgaard M., Rasmussen J., Christensen S.T., Surma E., DE ZEE M., Analysis of musculoskeletal systems in the AnyBody Modeling System, Simul. Model. Pract. Theory, 2006, 14, 1100-1111.

[6] Hayden A.M., Hayes A.M., Brechbuhler J.L., IsRael H., PlaCE H.M., The effect of pelvic motion on spinopelvic parameters, Spine J., 2018, 18, 173-178.

[7] Joszko K., GziK M., WolańSKi W., GZIK-Zroska B., KAWLEWSKA E., Biomechanical evaluation of human lumbar spine in spondylolisthesis, J. Appl. Biomed., 2018, 16, 51-58.

[8] JuRKojć J., Michnik R., JolanTa P., Identification of muscle forces acting in lower limbs with the use of planar and spatial mathematical model, J. Vibroengineering, 2009, 11, 566-570.

[9] Koszela K., KRUKOwSKa S., WoldaŃSKA-OKOŃSKA M., Back pain as a lifestyle disease, Pediatr. i Med. Rodz., 2017, 13, 344-351.

[10] Laird R.A., Gilbert J., Kent P., Keating J.L., Comparing lumbo-pelvic kinematics in people with and without back pain: a systematic review and meta-analysis, BMC Musculoskelet. Disord., 2014, 15, 229.

[11] LeVine D., Whittle M.W., The effects of pelvic movement on lumbar lordosis in the standing position, J. Orthop. Sports Phys. Ther., 1996, 24, 130-135.

[12] McGRegor A.H., Hukins D.W.L., Lower limb involvement in spinal function and low back pain, J. Back Musculoskelet. Rehabil., 2009, 22, 219-222.
[13] Murrie V.L., Dixon A.K., Hollingworth W., Wilson H., DOYLE T.A.C., Lumbar lordosis: Study of patients with and without low back pain, Clin. Anat., 2003, 16, 144-147.

[14] Nowakowska K., Gzik M., Michnik R., Myśliwiec A., JuRKOJĆ J., SuchoŃ S. et al., The Loads Acting on Lumbar Spine During Sitting Down and Standing Up, [in:] M. Gzik, E.Tkacz, Z. Paszenda, E. Piętka (Eds.), Innovations in Biomedical Engineering. Advances in Intelligent Systems and Computing, Springer, Cham, 2017, 169-176.

[15] Nowakowska K., Michnik R., Myśliwiec A., Chrzan M., Impact of strengthening of the erector spinae muscle on the values of loads of the muskuloskeletal system in the lumbar spine section, Engineering Mechanics, 2017, 23rd International Conference May 15-18, 2017, Svratka, Czech Republic, Book of full tests, Ed. Vladimir Fuis, 718-721.

[16] Nowakowska K., Michnik R., Myśliwiec A., Zadoń H., Identification of loads occurring in the lumbar spine section during the action of lifting objects, Engineering Mechanics, 2018, 24th International Conference, May 14-17, 2018, Svratka, Czech Republic, 2018, 617-620.

[17] Pries E., Dreischarf M., Bashkuev M., Putzier M., SCHMIDT H., The effects of age and gender on the lumbopelvic rhythm in the sagittal plane in 309 subjects, J. Biomech., 2015, 48, 3080-3087.

[18] Raciborski F., Gasik R., KTAK A., Disorders of the spine. A major health and social problem, Reumatologia, 2016, 54, 196-200.

[19] Searle A., Spink M., Ho A., ChUTER V., Exercise interventions for the treatment of chronic low back pain: A systematic review and meta-analysis of randomised controlled trials, Clin. Rehabil., 2015, 29, 1155-1167.

[20] Shum G.L.K., Crosbie J., Lee R.Y.W., Effect of Low Back Pain on the Kinematics and Joint Coordination of the Lumbar Spine and Hip During Sit-to-stand and Stand-to-sit, Spine (Phila. Pa. 1976), 2005, 30, 1998-2004.

[21] SzkodA-Poliszuk K., ŻAK M., Pezowicz C., Finite element analysis of the influence of three-joint spinal complex on the change of the intervertebral disc bulge and height, Int. J. Numer. Method. Biomed. Eng., 2018, 34 (9), e3107.

[22] Walker M.L., Rothstein J.M., FinUCANE S.D., Lamb R.L., Relationships between lumbar lordosis, pelvic tilt, and abdominal muscle performance, Phys. Ther., 1987, 67, 512-516.

[23] Wong K.W.N., LuK K.D.K., LeONG J.C.Y., Wong S.F., Wong K.K.Y., Continuous Dynamic Spinal Motion Analysis, Spine (Phila. Pa. 1976), 2006, 31, 414-419.

[24] Zadoń H., Michnik R., Nowakowska K., Mrśliwiec A., Assessment of Loads Exerted on the Lumbar Segment of the Vertebral Column in Everyday-Life Activities - Application of Methods of Mathematical Modelling, [in:] E. Pietka, P. Badura, J. Kawa, W. Wieclawek (Eds.), Information Technology in Biomedicine. ITIB 2019. Advances in Intelligent Systems and Computing, Springer, Cham, 2019, 554-565.

[25] De Zee M., Hansen L., Wong C., Rasmussen J., Simonsen E.B., A generic detailed rigid-body lumbar spine model, J. Biomech., 2007, 40, 1219-1227.

[26] Zuk M., Syczewska M., Pezowicz C., Influence of uncertainty in selected musculoskeletal model parameters on muscle forces estimated in inverse dynamics-based static optimization and hybrid approach, J. Biomech. Eng., 2018, 140.

[27] Dane statystyczne dot. absencji chorobowych w roku 2018 opublikowane przez ZUS (Statistical data about sickness absence published by the National Social Insurance Office (ZUS)), Information Note, (2018) (in Polish). 